\title{
Bacterial
}

\section{Microcompartment-Dependent 1,2-Propanediol Utilization of Propionibacterium freudenreichii}

\author{
Alexander Dank ${ }^{1 \dagger}$, Zhe Zeng ${ }^{1 \dagger}$, Sjef Boeren ${ }^{2}$, Richard A. Notebaart ${ }^{1}$, Eddy J. Smid ${ }^{1 *}$ and \\ Tjakko Abee ${ }^{\text {** }}$ \\ ${ }^{1}$ Food Microbiology, Wageningen University and Research, Wageningen, Netherlands, ${ }^{2}$ Laboratory of Biochemistry, \\ Wageningen University and Research, Wageningen, Netherlands
}

OPEN ACCESS

Edited by:

Ulrike Kappler,

The University of Queensland,

Australia

Reviewed by:

Chiranjit Chowdhury,

National Chemical Laboratory (CSIR),

India

Bhawani Chamlagain

University of Helsinki, Finland

*Correspondence:

Tjakko Abee

tjakko.abee@wur.n

Eddy J. Smid

eddy.smid@wur.nl

${ }^{\dagger}$ These authors have contributed equally to this work and share first

authorship

Specialty section:

This article was submitted to Microbial Physiology and Metabolism,

a section of the journal

Frontiers in Microbiology

Received: 12 March 2021

Accepted: 16 April 2021

Published: 12 May 2021

Citation:

Dank A, Zeng Z, Boeren S, Notebaart RA, Smid EJ and Abee T

(2021) Bacterial

Microcompartment-Dependent

1,2-Propanediol Utilization of

Propionibacterium freudenreichii.

Front. Microbiol. 12:679827.

doi: 10.3389/fmicb.2021.679827
Bacterial microcompartments (BMCs) are proteinaceous prokaryotic organelles that enable the utilization of substrates such as 1,2-propanediol and ethanolamine. BMCs are mostly linked to the survival of particular pathogenic bacteria by providing a growth advantage through utilization of 1,2-propanediol and ethanolamine which are abundantly present in the human gut. Although a 1,2-propanediol utilization cluster was found in the probiotic bacterium Propionibacterium freudenreichii, BMC-mediated metabolism of 1,2-propanediol has not been demonstrated experimentally in $P$. freudenreichii. In this study we show that $P$. freudenreichii DSM 20271 metabolizes 1,2-propanediol in anaerobic conditions to propionate and 1-propanol. Furthermore, 1,2-propanediol induced the formation of BMCs, which were visualized by transmission electron microscopy and resembled BMCs found in other bacteria. Proteomic analysis of 1,2-propanediol grown cells compared to L-lactate grown cells showed significant upregulation of proteins involved in propanediol-utilization (pdu-cluster), DNA repair mechanisms and BMC shell proteins while proteins involved in oxidative phosphorylation were down-regulated. 1,2-Propanediol utilizing cells actively produced vitamin $\mathrm{B}_{12}$ (cobalamin) in similar amounts as cells growing on L-lactate. The ability to metabolize 1,2-propanediol may have implications for human gut colonization and modulation, and can potentially aid in delivering propionate and vitamin $\mathrm{B}_{12}$ in situ.

Keywords: bacterial microcompartments, Propionibacterium freudenreichii, vitamin $\mathrm{B}_{12}$, metabolosome, propanediol utilization cluster, probiotic, intestine

\section{INTRODUCTION}

Propionibacterium freudenreichii is a Gram-positive, non-spore forming bacterium which has been linked to several potential health promoting effects, such as reducing intestinal inflammation, immunomodulation, modulation of intestinal motility and absorption, reduction of pathogen adhesion and enhancement of bifidobacteria [reviewed by Cousin et al. (2011)]. P. freudenreichii is able to cope with a large variety of stresses (oxidative, bile salt, and temperature; Falentin et al., 2010) and consequently has good survival capabilities in the upper intestinal tract (Huang and Adams, 2004). Survival of $P$. freudenreichii in the intestinal environment is supported by expression 
of pathways involved in the metabolism of substrates present in the intestinal environment, such as propanediol (Saraoui et al., 2013).

1,2-propanediol is a major end product from anaerobic degradation of rhamnose or fucose by the human intestinal microbiota and serves as an important carbon source for 1,2-propanediol utilizing bacteria, which can metabolize 1,2propanediol into propionate, generating ATP, and into 1propanol for maintaining redox balance. The metabolism of 1,2propanediol produces the toxic intermediate propionaldehyde (Sampson and Bobik, 2008). Some bacteria can protect themselves from toxic intermediates by encapsulating the enzymatic processes in self-assembling proteinaceous organelles called bacterial microcompartments (BMCs; Axen et al., 2014). BMCs are typically about $40-200 \mathrm{~nm}$ in diameter and are made of three types of shell proteins: hexamers, pseudohexamers, and pentamers (Kerfeld et al., 2018). Based on the highly conserved domain of shell proteins, Axen et al. (2014) predicted the presence of these organelles in 23 different bacterial phyla, including Actinobacteria which includes the species $P$. freudenreichii. However, no experimental evidence of BMC mediated utilization of 1,2-propanediol has been shown in $P$. freudenreichii and its role as carbon source is yet to be elucidated.

Bacterial microcompartment-mediated catabolism of substrates involving a toxic aldehyde intermediate is driven by three core enzymes: aldehyde dehydrogenase, alcohol dehydrogenase, and phosphotransacylase (Axen et al., 2014). The signature enzyme in the propanediol utilization $(p d u)$ pathway is propanediol dehydratase, a vitamin $\mathrm{B}_{12}$-dependent enzyme catalyzing the reaction of 1,2-propanediol to propionaldehyde (Cheng et al., 2011; Axen et al., 2014). Vitamin $B_{12}$ is produced in high quantities by $P$. freudenreichii (Burgess et al., 2009) and it also requires vitamin $\mathrm{B}_{12}$ as a cofactor for a key enzyme in its characteristic Wood-Werkman cycle. It is suggested that Actinobacteria such as $P$. freudenreichii obtained the $p d u$ cluster through horizontal gene transfer from Clostridiales (Ravcheev et al., 2019), in which acquisition may have been supported by the ability of $P$. freudenreichii to produce vitamin $\mathrm{B}_{12}$ de-novo (Roessner et al., 2002). BMCs are also found in some pathogenic bacteria, such as Salmonella enterica, Enterococcus faecalis, Listeria monocytogenes, pathogenic Escherichia coli, and Clostridium perfringens (Kerfeld et al., 2018). BMC-mediated utilization of 1,2-propanediol increases competitive fitness of pathogens in the gut and consequently has been linked to virulence (Jakobson and Tullman-Ercek, 2016). However, symbiotic relationships depending on 1,2-propanediol metabolism have also been shown for beneficial Lactobacillus reuteri and Bifidobacterium breve (Cheng et al., 2020). The ability to degrade 1,2-propanediol may have similar implications for the bifidogenic capacity reported for P. freudenreichii (Kaneko et al., 1994) and consequences for gut modulation and competition with pathogenic bacteria. Furthermore, the active production of vitamin $\mathrm{B}_{12}$ during metabolism of 1,2-propanediol has not been studied yet in P. freudenreichii.

In this study we present evidence for BMC-mediated anaerobic growth of $P$. freudenreichii on 1,2-propanediol, evidenced by substrate utilization, propionate and 1-propanol production, and vitamin $\mathrm{B}_{12}$ synthesis. Using transmission electron microscopy (TEM) and proteomics, we confirmed the presence of BMCs, Pdu BMC shell proteins and enzymes in pdu-induced $P$. freudenreichii.

\section{MATERIALS AND METHODS}

\section{Strains, Culture Conditions, and Growth Measurement}

Propionibacterium freudenreichii DSM 20271 was grown anaerobically (Anoxomat modified atmosphere, MART; 10\% $\mathrm{CO}_{2}, 5 \% \mathrm{H}_{2}$, and $85 \% \mathrm{~N}_{2}$ ) at $30^{\circ} \mathrm{C}$ in $50 \mathrm{~mL}$ tubes containing $40 \mathrm{~mL}$ complex media containing per liter: $10 \mathrm{~g}$ tryptone, $5 \mathrm{~g}$ yeast extract, and $5 \mathrm{~g} \mathrm{KH}_{2} \mathrm{PO}_{4}$ supplemented with $100 \mathrm{mM}$ L-lactate or 1,2-propanediol. All media was set at $\mathrm{pH} 7.0$ by addition of $5 \mathrm{M} \mathrm{NaOH}$ and was filter sterilized through $0.2 \mu \mathrm{m}$ filters into sterile flasks. $\mathrm{OD}_{600}$ measurements and extracellular metabolite samples were taken daily for a time period of 7 days.

\section{Analysis of Extracellular Metabolites Using High Performance Liquid Chromatography}

Culture samples were taken at various time intervals and were analyzed for extracellular metabolites by high performance liquid chromatography. $1 \mathrm{~mL}$ culture was centrifuged at $17,000 \times g$ for $1 \mathrm{~min}$ and the supernatant was collected. $0.5 \mathrm{~mL}$ supernatant was treated with $0.25 \mathrm{~mL}$ Carrez A and $0.25 \mathrm{~mL}$ Carrez B, vortexed and centrifuged at $17,000 \times g$ for $2 \mathrm{~min}$. $200 \mu \mathrm{L}$ supernatant was stored in HPLC vials at $-20^{\circ} \mathrm{C}$ upon analysis. HPLC was performed as described by Zeng et al. (2019). Quantification was performed by addition of a standard curve containing L-lactate, acetate, propionate, 1,2-propanediol, and 1-propanol.

\section{Transmission Electron Microscopy}

Propionibacterium freudenreichii cultures were grown anaerobically at $30^{\circ} \mathrm{C}$ in $100 \mathrm{mM}$ L-lactate or 1,2-propanediol containing media. Samples were collected after 6 days of incubation (early stationary phase for 1,2-propanediol-grown cells). About $10 \mu \mathrm{g}$ of dry cell biomass was fixed for $2 \mathrm{~h}$ in $2.5 \%$ (v/v) glutaraldehyde in $0.1 \mathrm{M}$ sodium cacodylate buffer $(\mathrm{pH}$ 7.2). After rinsing in the same buffer, a post-fixation was done in $1 \%(\mathrm{w} / \mathrm{v}) \mathrm{OsO}_{4}$ for $1 \mathrm{~h}$ at room temperature. The samples were dehydrated using ethanol. The dehydrated cell pellets were then embedded in resin (Spurr HM20) for $10 \mathrm{~h}$ at $70^{\circ} \mathrm{C}$. Thin sections $(<100 \mathrm{~nm})$ of polymerized resin samples were obtained with microtomes. After staining with $2 \%(\mathrm{w} / \mathrm{v})$ aqueous uranyl acetate, the samples were analyzed with a Jeol 1400 plus TEM with $120 \mathrm{kV}$ setting as described by Zeng et al. (2019).

\section{Vitamin $\mathbf{B}_{\mathbf{1 2}}$ Quantification}

After 7 days of incubation the vitamin $B_{12}$ (cobalamin) concentration was determined using a microbiological assay (Vitafast vitamin $\mathrm{B}_{12}$ kit, R-biopharm) for $P$. freudenreichii grown in $66 \mathrm{mM}$ L-lactate and $49 \mathrm{mM}$ 1,2-propanediol medium. 
Briefly, $1 \mathrm{~mL}$ of culture was disrupted by bead beating (lysing matrix B, mp-bio) $2 \times 1 \mathrm{~min}$ at $4.5 \mathrm{~m} / \mathrm{s}$ with $1 \mathrm{~min}$ on ice in between. Samples were centrifuged and diluted with water to appropriate concentrations for the test kit and were heatextracted for $30 \mathrm{~min}$ at $95^{\circ} \mathrm{C}$. The samples were cooled to room temperature and vitamin $\mathrm{B}_{12}$ detection was performed according to the manufacturer protocol.

\section{Proteomics}

Propionibacterium freudenreichii cells were cultured in media supplemented with $100 \mathrm{mM}$ L-lactate or $100 \mathrm{mM} \mathrm{1,2-propanediol}$ for 7 days. Cell pellets were harvested by centrifugation of $1 \mathrm{~mL}$ of sample at $17,000 \times g$ for $1 \mathrm{~min}$ in table top centrifuges and cell pellets were frozen at -80 degrees. Samples were washed twice with $100 \mathrm{mM}$ Tris (pH 8) and resuspended in $100 \mu 1100 \mathrm{mM}$ Tris. Samples were lysed by sonication for $45 \mathrm{~s}$ twice while cooling 1 min on ice. Protein content was determined using Pierce Coomassie protein assay and samples were diluted to $1 \mu \mathrm{g} / \mu \mathrm{l}$ using Tris- $\mathrm{HCl} \mathrm{pH} \mathrm{8.} \mathrm{Samples} \mathrm{were} \mathrm{prepared} \mathrm{according} \mathrm{to} \mathrm{the}$ filter assisted sample preparation protocol (FASP; Wiśniewski et al., 2009) with the following steps: reduction with $15 \mathrm{mM}$ dithiothreitol, alkylation with $20 \mathrm{mM}$ acrylamide, and digestion with sequencing grade trypsin overnight. Each prepared peptide sample was analyzed by injecting $(18 \mu \mathrm{l})$ into a nanoLCMS/MS (Thermo nLC1000 connected to a LTQ-Orbitrap XL) as described previously (Lu et al., 2011). LCMS data with all MS/MS spectra were analyzed with the MaxQuant quantitative proteomics software package (Cox et al., 2014) as described before (Smaczniak et al., 2012; Wendrich et al., 2017).

A protein database with the protein sequence of P. freudenreichii DSM 20271 (ID:UP000032238) was downloaded from UniProt. Filtering and further bioinformatics and statistical analysis of the MaxQuant ProteinGroups file were performed with Perseus (Tyanova et al., 2016). Reverse hits and contaminants were filtered out. Protein groups were filtered to contain minimally two peptides for protein identification of which at least one is unique and at least one is unmodified. Also, each group required three valid values in at least one of the two experimental groups. A volcano plot was prepared based on the Student's $t$-test difference between samples. Volcano plots were produced in Rstudio using EnhancedVolcano (Blighe, 2018). Proteins were considered to be significantly different amongst sample if $p<0.05$ and 4 -fold change difference was detected. KEGG gene set enrichment analysis was performed using Clusterprofiler (Yu et al., 2012) and 2-fold change difference amongst proteins. The mass spectrometry proteomics data have been deposited to the ProteomeXchange Consortium via the PRIDE (Vizcaíno et al., 2016) partner repository with the dataset identifier PXD024700.

\section{Predicting BMCs Shell Proteins}

The Hidden Markov Models (HMMs) of two BMC shell protein domains listed as Pf00936 and Pf03319 were retrieved from the Pfam database to predict BMC shell proteins as described in Axen et al., 2014; Zeng et al., 2019. Shell proteins were predicted by a HMM search using the HMMER package and a local protein database of $P$. freudenreichii DSM 20271 genome [CP010341.1 (Deptula et al., 2017)]. All hits with an $e$-value less than or equal to 1e-05 that correspond to a genomic record from Genbank, RefSeq, EMBL, or DDBJ databases were accepted as BMC shell protein homologs (Supplementary File 1).

\section{RESULTS}

\section{Growth Performance and Metabolite Production on 1,2-Propanediol and L-Lactate}

Anaerobic growth of $P$. freudenreichii on $100 \mathrm{mM}$ 1,2propanediol was monitored for 6 days with daily sampling in triplicate. As a control, $P$. freudenreichii was cultured on

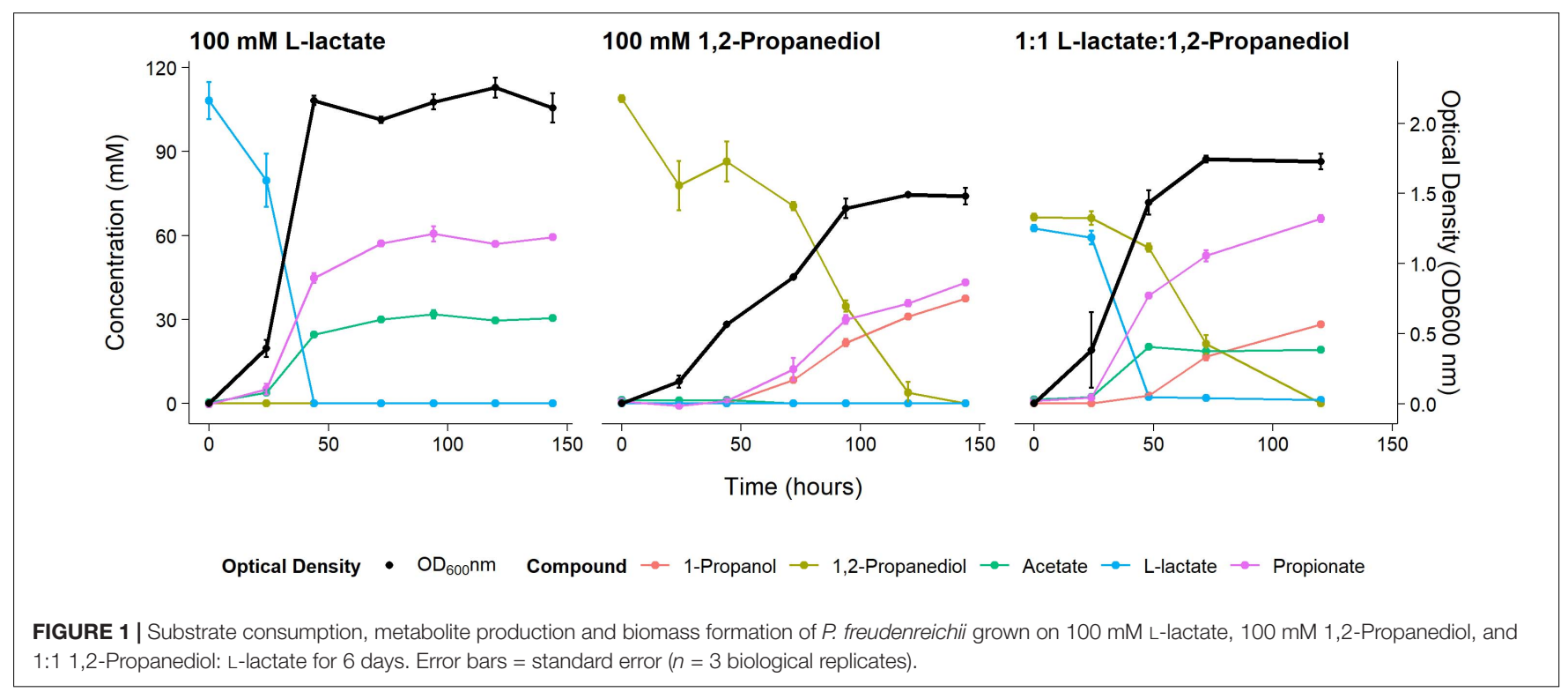


$100 \mathrm{mM}$ L-lactate. P. freudenreichii was able to completely metabolize $100 \mathrm{mM}$ 1,2-propanediol during a period of 6 days (see Figure 1). This resulted in the production of $37.5 \pm 0.1 \mathrm{mM}$ 1-propanol and $43.2 \pm 0.5 \mathrm{mM}$ propionate. No production of acetate was observed, and with the current HPLC method used, approximately $28 \mathrm{mM}$ of expected $\mathrm{C}_{3}$ compounds is missing, conceivably volatile propionaldehyde. In the control cultures, $100 \mathrm{mM}$ L-lactate was consumed within 2 days, resulting in $65.1+0.1 \mathrm{mM}$ propionate and $30.5 \pm 0.1 \mathrm{mM}$ acetate, close the expected molar ratio of 2:1 (Seeliger et al., 2002). Growth on 1,2propanediol resulted in slower growth, with a stationary phase after $120 \mathrm{~h}$ vs $48 \mathrm{~h}$ in L-lactate. Our results clearly demonstrate that $P$. freudenreichii can grow on 1,2-propanediol, metabolizing it to propionate and 1-propanol.

To monitor metabolism of 1,2-propanediol in the presence of other carbon sources $P$. freudenreichii was grown in media containing both L-lactate and 1,2-propanediol. When $\mathrm{NAD}^{+} / \mathrm{NADH}$ pools are shared between the cytosol and the BMC as discussed by Ferlez et al. (2019), the highest ATP yield from 1,2-propanediol in the presence of L-lactate can be obtained by co-fermenting 1,2-propanediol and L-lactate solely to propionate (see Supplementary Text 1). The expected ATP yield with L-lactate and 1,2-propanediol as mixed substrates is higher compared to cells growing solely on L-lactate (see Supplementary Text 1). We found production of $67.1 \pm 1.5 \mathrm{mM}$ propionate, $19.0 \pm 1.0 \mathrm{mM}$ of acetate, and $28.3 \pm 2.8 \mathrm{mM}$ 1-propanol in cells growing on the mixed substrates. Assuming 1-propanol:propionate is produced in a $1: 1$ ratio from 1,2propanediol and acetate:propionate is produced in a 1:2 ratio from L-lactate, $28 \mathrm{mM}$ of the propionate originates from the 1,2propanediol metabolism and $38 \mathrm{mM}$ propionate originates from L-lactate metabolism. This matches the total found propionate of $67.1 \pm 1.5 \mathrm{mM}$ propionate in the samples. In mixed substrate conditions the amounts of total products formed indicate no apparent loss of $\mathrm{C}_{3}$ compounds, which may indicate reduced loss of volatile propionaldehyde. Biomass formation with 1:1 L-lactate:1,2-propanediol-grown cells was found to be lower compared to $100 \mathrm{mM}$ L-lactate-grown cells. These results indicate no apparent energetic benefit from mixed substrate conditions compared to mono substrate conditions, pointing toward independent pathways. Our results show mixedsubstrate metabolism influences the total amount of shortchain fatty acid production, but pathway interactions are not apparent.

\section{Vitamin $\mathbf{B}_{\mathbf{1 2}}$ Production}

To demonstrate active vitamin $B_{12}$ production under 1,2propanediol utilizing conditions, $P$. freudenreichii was grown for 7 days in either yeast extract medium supplemented with $66 \mathrm{mM}$ L-lactate or $49 \mathrm{mM}$ 1,2-propanediol. Biomass formation, substrate utilization, and vitamin $\mathrm{B}_{12}$ production by $P$. freudenreichii was monitored after 7 days of incubation.
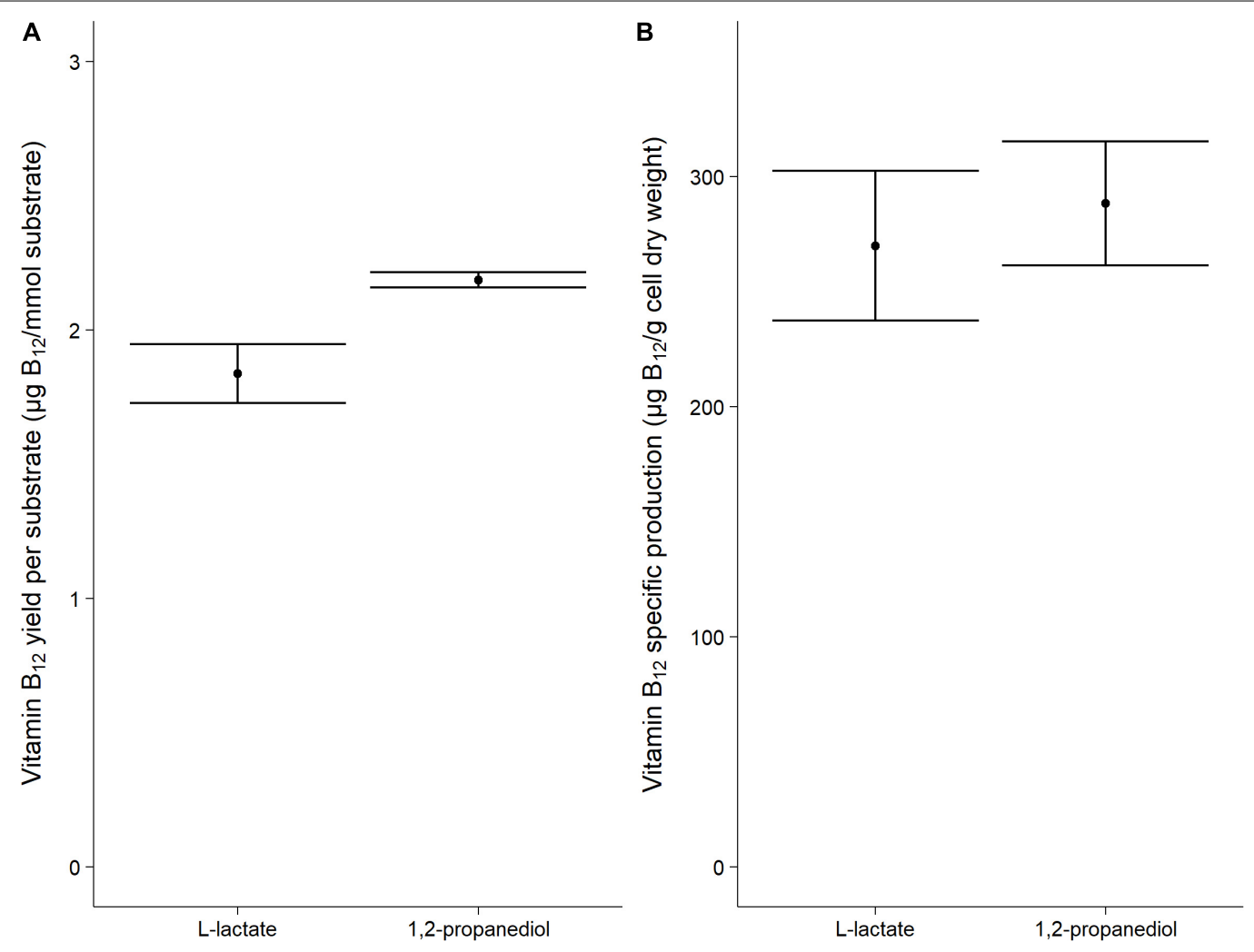

FIGURE 2 | Vitamin $B_{12}$ formation in cells grown on L-lactate or 1,2-propanediol. (A) vitamin $B_{12}$ yield in $\mu \mathrm{g} / \mathrm{mmol}$ substrate (B) Biomass specific vitamin $B_{12}$ production in $\mu \mathrm{g} / \mathrm{g}$ cell dry weight. Error bars $=$ standard error $(n=3$ biological replicates). 
Incubation vessels were not opened in-between to prevent side effects by oxygen-dependent stimulation of vitamin $\mathrm{B}_{12}$ production, as has been shown for P. freudenreichii (QuesadaChanto et al., 1998). Again, complete utilization of L-lactate and 1,2-propanediol was found (Supplementary File 2).

Vitamin $\mathrm{B}_{12}$ was produced both when grown on L-lactate as on 1,2-propanediol $(1.84 \pm 0.11 \mu \mathrm{g} / \mathrm{mmol} \mathrm{L-lactate} \mathrm{vs}$ $2.19 \pm 0.03 \mu \mathrm{g} / \mathrm{mmol}$ 1,2-propanediol). The specific vitamin $\mathrm{B}_{12}$ production when grown on L-lactate and on 1,2-propanediol was $270 \pm 33 \mu \mathrm{g} / \mathrm{g}$ cells and $288 \pm 27 \mu \mathrm{g} / \mathrm{g}$ cells, respectively, (Figure 2). No significant difference was thus found for the production of vitamin $\mathrm{B}_{12}$ per $\mathrm{g}$ cells when growing on either L-lactate supplemented media or 1,2-propanediol supplemented media. In complex media, 1,2-propanediol as carbon source thus supports vitamin $\mathrm{B}_{12}$ production by $P$. freudenreichii.

\section{Proteomics and Electron Microscopy of L-Lactate and 1,2-Propanediol Grown Cells}

Cells growing in media supplemented with L-lactate (control) and with 1,2-propanediol were visualized using TEM. Thin sections of cells grown on 1,2-propanediol supplemented media clearly display cellular structures which were not found in the cells grown on L-lactate supplemented media (see Figure 3), and those structures resemble BMC structures found in other bacteria including L. monocytogenes (Zeng et al., 2019), S. enterica (Crowley et al., 2008), and E. coli (Toraya et al., 1979).

Proteome analysis revealed that BMC structural shell proteins (PduA, PduB, PduK, PduJ, PduN, and PduM) and enzymes involved in 1,2-propanediol utilization (PduL, PduC, PduD, PduE, PduP, PduO, and PduQ) were significantly more abundant in cells grown with 1,2-propanediol as a substrate compared to cells grown on L-lactate (Figure 4). Lactate permease, succinate dehydrogenase subunits A, C1 (SdhA, SdhC1) were found to be significantly more abundant in the L-lactate-grown cells compared to 1,2-propanediol-grown cells, indicating a decrease of proteins in lactate-degradation pathways such as the Wood-Werkman cycle. Gene set enrichment analysis of KEGG pathways (see Supplementary File 3) revealed upregulation of the propanediol degradation pathway in 1,2-propanediol grown cells compared to L-lactate grown cells (adjusted $p$-value $<0.10$ ). DNA repair mechanisms were also found to be activated in 1,2 -propanediol-grown cells (adjusted $p$-value $<0.10$ ). In 1,2propanediol-grown cells significant suppression of oxidative phosphorylation was found compared to L-lactate grown cells (adjusted $p$-value $<0.01$ ). The measured consumption of 1,2-propanediol, production of 1-propanol and propionate, visualized cellular structures, and strong upregulation of the $p d u$ loci proteins provide evidence for activation of BMC-dependent 1,2-pdu in P. freudenreichii DSM 20271.

\section{Overview of BMC-Dependent 1,2-Propanediol Metabolism Model in $P$ freudenreichii}

Subsequent analysis of the $p d u$ cluster in $P$. freudenreichii DSM 20271 identified two distant loci, with locus 1 starting from RM25_0852 to RM25_0857 and locus 2 starting from RM25_1258 to RM25_1273 (Figure 5A). Locus 1 contains four genes which are $p o c R$ encoding a transcriptional regulator, $p d u Q$ encoding 1-propanol dehydrogenase, $p d u V$ with unknown function and $p d u U$ encoding BMC shell protein. Locus 2 carries 14 genes including 6 genes encoding BMC shell proteins and 8 genes encoding enzymes for the 1,2-propanediol

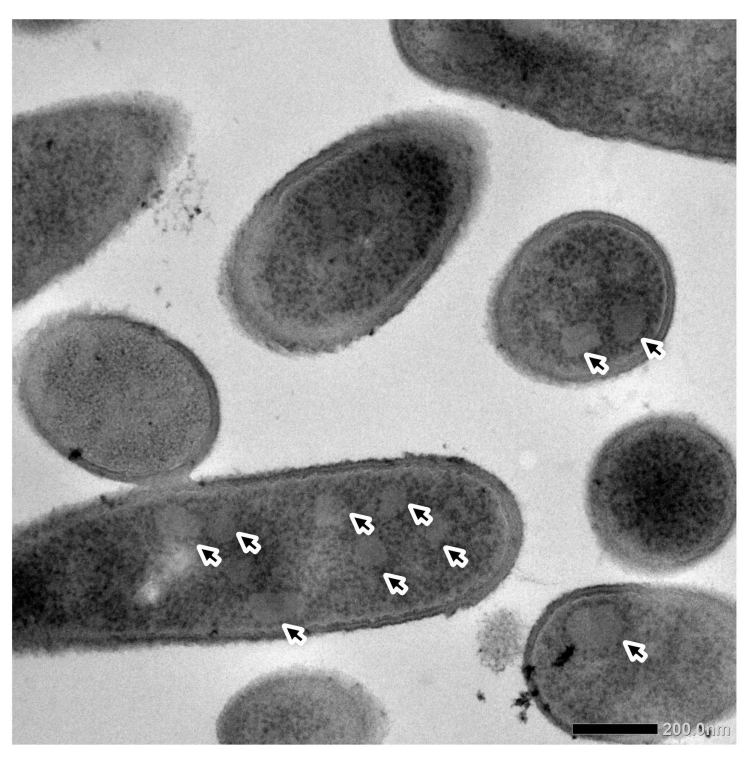

Grown in 1,2-Propanediol media

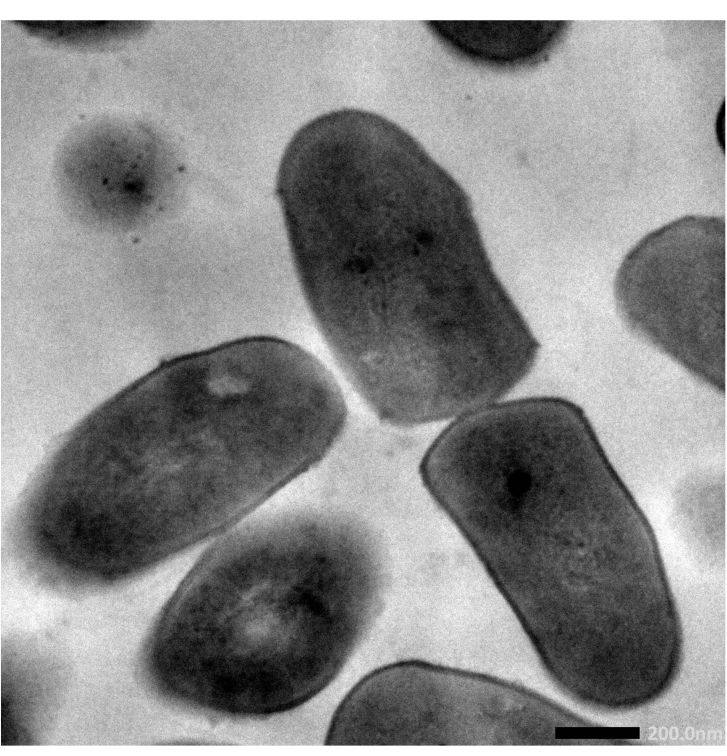

Grown in Lactate media

FIGURE 3 | Visualization of bacterial microcompartments in propanediol media (left) and absence in lactate media (right). 
degradation pathway and is not preceded by any known transcriptional regulators.

Based on the structural studies of BMCs (Sutter et al., 2017; Greening and Lithgow, 2020) and our understanding of 1,2-propanediol metabolism (Sampson and Bobik, 2008; Kerfeld et al., 2018; Zeng et al., 2019), we propose the model of BMC-dependent 1,2-propanediol metabolism in P. freudenreichii, with the predicted BMC shell proteins PduA, PduB, PduK, PduJ, PduM, PduN, and PduU constituting the self-organized icosahedral organelle (Kerfeld et al., 2018; Greening and Lithgow, 2020). As illustrated in Figure 5B, the catabolism of 1,2-propanediol starts with the conversion of 1,2-propanediol to propionaldehyde by vitamin $\mathrm{B}_{12}$-dependent diol dehydratase PduCDE. The toxic propionaldehyde is then converted to propionate by the enzyme CoA-dependent propionaldehyde dehydrogenase PduP, followed by action of phosphate propanoyltransferase PduL, and potential propionate kinase located in the cytoplasm, resulting in the end product propionate and the production of ATP. The other end product is produced following conversion of propionaldehyde by propanol dehydrogenase PduQ into 1-propanol. The diol dehydratase reactivase $\mathrm{PduGH}$ and corrinoid adenosyltransferase $\mathrm{PduO}$ are linked to the supply and recycling of vitamin $B_{12}$.

\section{DISCUSSION}

Propionibacterium freudenreichii is commonly found in the rumen and colon of animals and in the human intestine (Bryant, 1959). In these environments fucose and rhamnose are degraded to 1,2-propanediol by the present microbiota (Xue et al., 2008). We showed 1,2-propanediol can be further metabolized into propionate and 1-propanol by $P$. freudenreichii, thereby supporting anaerobic growth. Previously, a locus containing 15 genes involved in $p d u$ was detected in P. freudenreichii (Falentin et al., 2010). In vivo gene expression analysis showed the $p d u$ operon to be expressed in $P$. freudenreichii cells contained in the colon environment of a pig (Saraoui et al., 2013), pointing toward $p d u$ in intestinal environments. Interestingly, in this study we identified the $p d u$ cluster distributed in two different loci in

\section{Volcano}

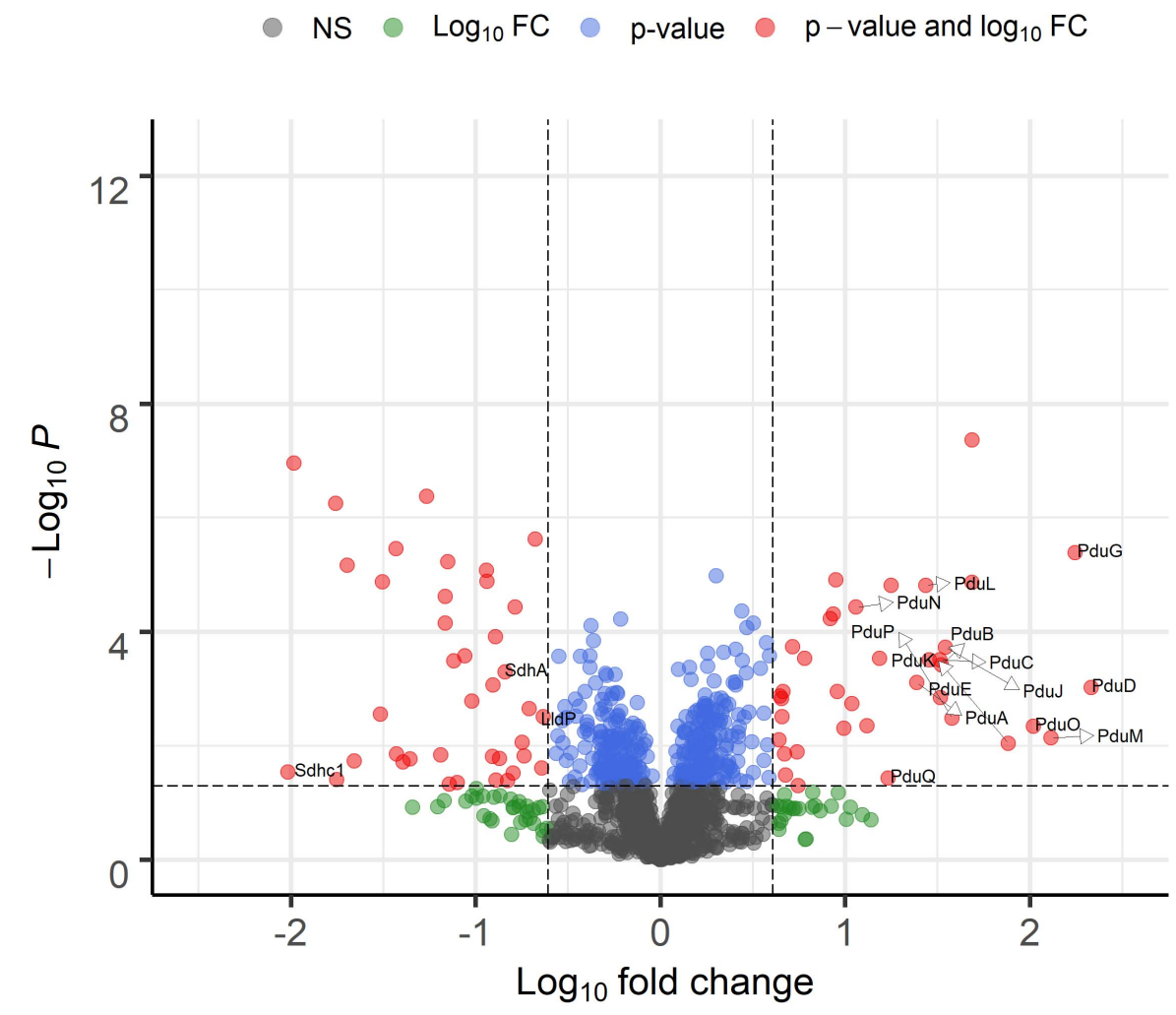

Total $=1398$ variables

FIGURE 4 | Volcano plot of proteomic analysis of cells grown in 1,2-propanediol media and L-lactate media. Positive $\log _{10}$ fold change indicate upregulation in 1,2 -propanediol-grown cells, negative $\log _{10}$ fold change indicate down regulation in 1,2-propanediol-grown cells compared to lactate-grown cells. Red dots indicate proteins with $p<0.05$ and 4 -fold difference expression. Blue dots indicate only $p<0.05$, green dots only 4-fold difference. Black dots indicate non-significant, non-differentially expressed proteins ( $n=3$ biological replicates). 
P. freudenreichii DSM 20271. The presence of the $p d u$ cluster seems to be species specific in propionic acid bacteria, as blasting the $p d u$ cluster of $P$. freudenreichii against Cutibacterium acnes, Acidipropionibacterium acidipropionici, A. thoenii, and A. jensenii did not result significant hits for key components in the cluster (see Supplementary File 4). Presence of 1,2propanediol in the medium induced expression of the two loci in P. freudenreichii DSM 20271 and resulted in BMC formation, 1,2-propanediol metabolism and consequently propionate and 1-propanol production. Our results show that expression of the $p d u$ cluster from two different loci results in effective BMC formation and 1,2-propanediol metabolism. Indeed, BMC genes are split into two or more loci in $40 \%$ of the prokaryotic genomes containing BMCs (Abdul-Rahman et al., 2013). In P. freudenreichii DSM 20271, locus 1 is preceded by transcriptional activator $p o c R$, which has been linked to activation of the $p d u$ cluster in Salmonella (Chen et al., 1994). We did not find any annotated transcriptional regulator in the vicinity of locus 2 . The presence of two different loci in many prokaryotic genomes suggests expression is controlled by additional regulators next to PocR. Indeed heterologous expression of BMCs uncoupled from their cognate transcriptional regulators has been reported previously (Wilson, 2021). The transcriptional regulation and activation of the two $p d u$ loci and the role of $p o c R$ in $P$. freudenreichii requires further attention.

Upregulation of the $p d u$ cluster and DNA repair mechanisms clearly indicated the crucial role of BMCs to protect $P$. freudenreichii from the toxic intermediate propionaldehyde produced in the degradation pathway. The ability to utilize substrates producing toxic intermediates upon degradation results in a competitive advantage to other gut microbiota (Jakobson and Tullman-Ercek, 2016), as shown for ethanolamine utilization by $S$. enterica during intestinal inflammation (Thiennimitr et al., 2011). Interestingly, the presence of genes encoding metabolosomes for the utilization of ethanolamine and propanediol has been linked to pathogenicity and aids in anaerobic growth and colonization of foodborne pathogens L. monocytogenes, C. perfringens, and S. typhimurium (Korbel et al., 2005; De Weirdt et al., 2012). It has been suggested for beneficial bacterium $L$. reuteri that competition for 1,2propanediol could result in decreased proliferation of pathogens
A

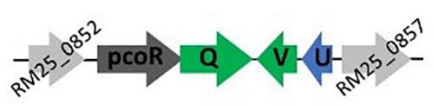

Locus 1
Propanediol Utilization Cluster (pdu)

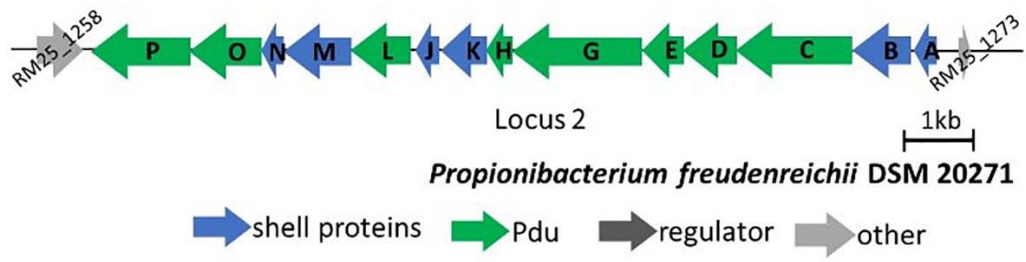

B BMC-dependent 1,2-Propanediol Metabolism Model

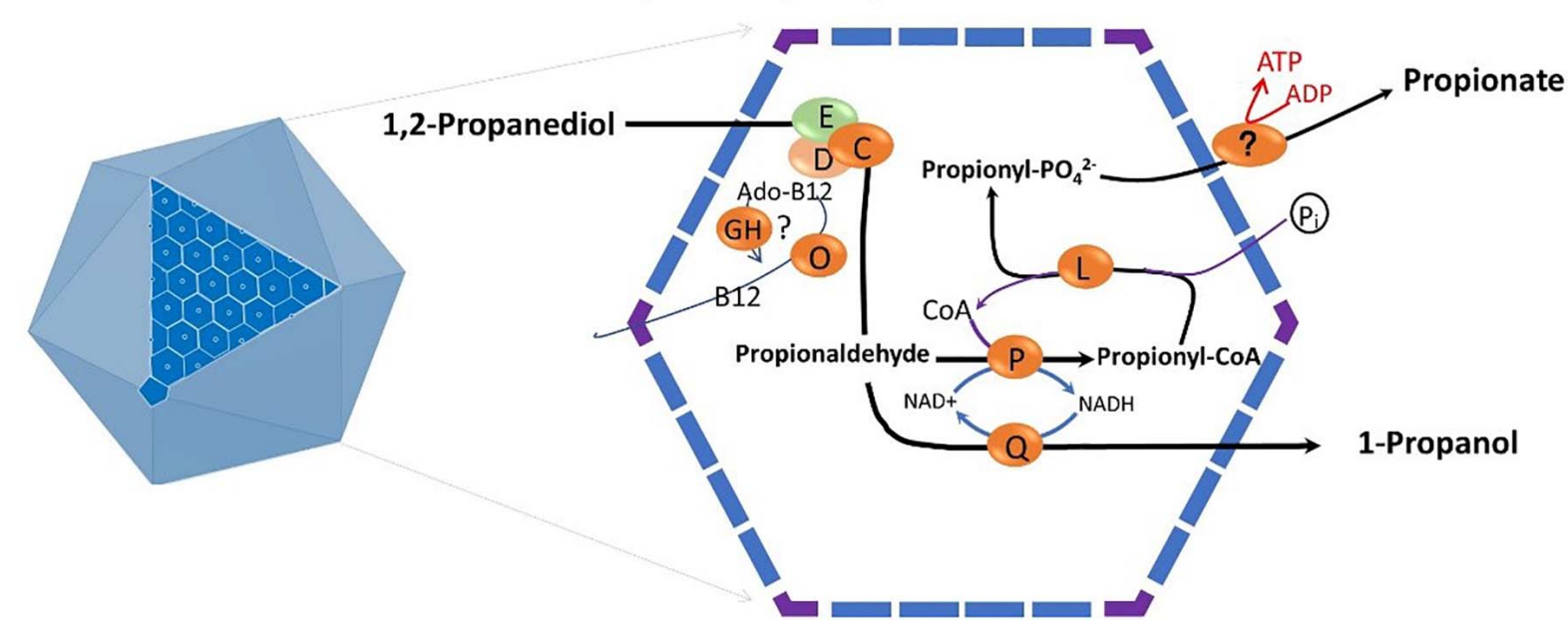

FIGURE 5 | (A) Analysis of the pdu gene cluster (Details in Supplementary File 1). Characters in orange represent Pdu enzymes, in blue represent BMC shell proteins, in dark represent regulator and in gray represent unannotated proteins with gene ID. (B) Model of BMC-dependent 1,2-propanediol metabolism. In the left, the icosahedral diagram represent BMC with one surface showing the assembly of shell proteins. In the right, the metabolic pathway of 1,2-propanediol metabolism. PduCDE, $\mathrm{B}_{12}$-dependent diol dehydratase; PduP, CoA-dependent propionaldehyde dehydrogenase; PduGH, diol dehydratase reactivase; PduO, corrinoid adenosyltransferase; PduS, Vitamin $\mathrm{B}_{12}$ reductase; PduL, phosphate propanoyltransferase; PduW, propionate kinase; and PduQ, propanol dehydrogenase. See text for details. 
(Cheng et al., 2020). P. freudenreichii is considered to be non-pathogenic and has the generally recognized as safe (GRAS) status (Meile et al., 2008). Substrate competition for 1,2-propanediol by $P$. freudenreichii may thus exert similar effects as suggested for $L$. reuteri on growth of pathogenic bacteria. This is in line with reports that $P$. freudenreichii can decrease adhesion of pathogens to human intestinal mucus cells (Collado et al., 2007). Furthermore, P. freudenreichii stimulates the growth of beneficial bifidobacteria (Satomi et al., 1999; Hojo et al., 2002) thereby promoting a healthy gut microbiota. The role of BMC-mediated 1,2-propanediol utilization by $P$. freudenreichii and its importance for modulating gut microbiota composition, both by substrate competition and promoting other beneficial microbiota, requires further investigation.

Our study shows the potential of $P$. freudenreichii to substantially contribute to the production of propionate in the human gut. Next to 1,2-propanediol, also lactic acid (Macfarlane and Gibson, 1997) is a major endproduct of microbial fermentation, which consequently can also be fermented by $P$. freudenreichii and additionally contributes to the production of propionate. In mixed substrate conditions both pathways remained active, albeit without apparent interaction based on metabolite formation. However, mixed substrate conditions decreased the loss of $\mathrm{C}_{3}$, suggesting less loss of volatile propionaldehyde, thus potentially more efficient BMC assembly. The assembly of BMCs in mixed substrate condition requires further attention. Propionate is linked to many putative health effects [reviewed by (Hosseini et al., 2011)] and can further stimulate bifidobacteria (Kaneko et al., 1994). The production of propionate from 1,2-propanediol further supports the potential of $P$. freudenreichii as a probiotic.

The metabolism of 1,2-propanediol requires propanediol dehydratase, which is vitamin $\mathrm{B}_{12}$-dependant (Axen et al., 2014). Vitamin $B_{12}$ is actively produced by $P$. freudenreichii as it is essential cofactor in a key enzyme in the WoodWerkman cycle, methylmalonyl-CoA mutase. Synthesis of vitamin $\mathrm{B}_{12}$ in $P$. freudenreichii follows the anaerobic pathway (Roessner et al., 2002), enabling production of vitamin $B_{12}$ for anaerobic metabolism of lactate. Here we show vitamin $\mathrm{B}_{12}$ is produced in similar amounts in complex medium when cells metabolize 1,2-propanediol or L-lactate as carbon source. In S. typhimurium pocR mediated expression of vitamin $\mathrm{B}_{12}$ is induced in the presence of propanediol (RichterDahlfors et al., 1994). We also identified pocR upstream of $p d u$ loci 1 in $P$. freudenreichii DSM 20271, but as discussed before its exact regulatory role in $P$. freudenreichii remains to be elucidated. Vitamin $B_{12}$ production is also regulated by a vitamin $B_{12}$ regulated riboswitch in $P$. freudenreichii. In Propionibacterium strain UT1 expression of vitamin $\mathrm{B}_{12}$ biosynthesis occurred at vitamin $\mathrm{B}_{12}$ concentrations of $750 \mu \mathrm{M}$, much higher compared to the vitamin $\mathrm{B}_{12}$ concentrations found in this study $(\sim 90 \mathrm{nM})$. The role of this riboswitch for vitamin $\mathrm{B}_{12}$ production during metabolism of 1,2-propanediol also remains to be elucidated. Based on our findings, we hypothesize that $P$. freudenreichii occupies a lactate and propanediol-rich niche in the gut environment. Symbiotic relationships have been shown for the production of vitamin $\mathrm{B}_{12}$ (Belzer et al., 2017; Sokolovskaya et al., 2020), which may be hard-wired in the vitamin $B_{12}$ production of $P$. freudenreichii. As next to vitamin $\mathrm{B}_{12}$ dependent lactate metabolism, BMC-mediated 1,2-propanediol metabolism supports anaerobic growth of $P$. freudenreichii, thereby contributing to in situ vitamin $\mathrm{B}_{12}$ production in the gut.

This study presents evidence for BMC-mediated vitamin $\mathrm{B}_{12}$ dependent utilization of 1,2-propanediol in P. freudenreichii. We have shown that 1,2-propanediol supports anaerobic growth of $P$. freudenreichii. It is conceivable that utilization of 1,2-propanediol could aid colonization of Propionibacterium freudenreichii in the human gut to exert beneficial effects, such as delivering vitamin $\mathrm{B}_{12}$ and propionate in situ, but these aspects require further study.

\section{DATA AVAILABILITY STATEMENT}

The original contributions presented in the study are publicly available. This data can be found here: https://www.ebi.ac.uk/ pride/archive/projects/PXD024700.

\section{AUTHOR CONTRIBUTIONS}

$A D$ and $Z Z$ designed and performed the experiments. SB, $A D$, and $Z Z$ performed proteomics and analyzed data. $Z Z$, $\mathrm{AD}, \mathrm{ES}, \mathrm{RN}$, and TA analyzed data. $\mathrm{ZZ}, \mathrm{AD}, \mathrm{ES}$, and TA wrote the manuscript. All authors read, edited and approved the manuscript.

\section{FUNDING}

The authors declare that this study received funding from Arla Foods (Denmark). The funder was not involved in the study design, collection, analysis, interpretation of data, the writing of this article or the decision to submit it for publication. ZZ was supported by a grant from the China Scholarship Council.

\section{ACKNOWLEDGMENTS}

We would like to thank Wageningen Electron Microscopy Centre (WEMC) for the use of their transmission electron microscope and for their help in sample preparation.

\section{SUPPLEMENTARY MATERIAL}

The Supplementary Material for this article can be found online at: https://www.frontiersin.org/articles/10.3389/fmicb.2021. 679827/full\#supplementary-material 


\section{REFERENCES}

Abdul-Rahman, F., Petit, E., and Blanchard, J. L. (2013). The distribution of polyhedral bacterial microcompartments suggests frequent horizontal transfer and operon reassembly. J. Phylog. Evol. Biol. 1:118.

Axen, S. D., Erbilgin, O., and Kerfeld, C. A. (2014). A taxonomy of bacterial microcompartment loci constructed by a novel scoring method. PLoS Comput. Biol. 10:e1003898. doi: 10.1371/journal.pcbi.1003898

Belzer, C., Chia, L. W., Aalvink, S., Chamlagain, B., Piironen, V., Knol, J., et al. (2017). Microbial metabolic networks at the mucus layer lead to dietindependent butyrate and Vitamin B12 production by intestinal symbionts. mBio 8:e00770-17. doi: 10.1128/mBio.00770-17

Blighe, K. (2018). Enhancedvolcano: Publication-Ready Volcano Plots With Enhanced Colouring and Labeling. Vienna: R Foundation for Statistical Computing.

Bryant, M. P. (1959). Bacterial species of the rumen. Bacteriol. Rev. 23, 125-153. doi: 10.1128/br.23.3.125-153.1959

Burgess, C. M., Smid, E. J., and van Sinderen, D. (2009). Bacterial vitamin B2, B11 and B12 overproduction: an overview. Int. J. Food Microbiol. 133, 1-7. doi: 10.1016/j.ijfoodmicro.2009.04.012

Chen, P., Andersson, D. I., and Roth, J. R. (1994). The control region of the $\mathrm{pdu} / \mathrm{cob}$ regulon in Salmonella typhimurium. J. Bacteriol. 176, 5474-5482. doi: 10.1128/jb.176.17.5474-5482.1994

Cheng, C. C., Duar, R. M., Lin, X., Perez-Munoz, M. E., Tollenaar, S., Oh, J. H., et al. (2020). Ecological importance of cross-feeding of the intermediate metabolite 1,2-propanediol between bacterial gut symbionts. Appl. Environ. Microbiol. 86, e190-e120. doi: 10.1128/AEM.00190-20

Cheng, S., Sinha, S., Fan, C., Liu, Y., and Bobik, T. A. (2011). Genetic analysis of the protein shell of the microcompartments involved in coenzyme B12-dependent 1, 2-propanediol degradation by Salmonella. J. Bacteriol. 193, 1385-1392. doi: 10.1128/jb.01473-10

Collado, M. C., Meriluoto, J., and Salminen, S. (2007). In vitro analysis of probiotic strain combinations to inhibit pathogen adhesion to human intestinal mucus. Food Res. Int. 40, 629-636. doi: 10.1016/j.foodres.2006.11.007

Cousin, F. J., Mater, D. D., Foligné, B., and Jan, G. (2011). Dairy propionibacteria as human probiotics: a review of recent evidence. Dairy Sci. Technol. 91, 1-26.

Cox, J., Hein, M. Y., Luber, C. A., Paron, I., Nagaraj, N., and Mann, M. (2014). Accurate proteome-wide label-free quantification by delayed normalization and maximal peptide ratio extraction, termed MaxLFQ. Mol. Cell. Prot. 13, 2513-2526. doi: 10.1074/mcp.m113.031591

Crowley, C. S., Sawaya, M. R., Bobik, T. A., and Yeates, T. O. (2008). Structure of the PduU shell protein from the Pdu microcompartment of Salmonella. Structure 16, 1324-1332. doi: 10.1016/j.str.2008.05.013

De Weirdt, R., Crabbe, A., Roos, S., Vollenweider, S., Lacroix, C., van Pijkeren, J. P., et al. (2012). Glycerol supplementation enhances L. reuteri's protective effect against S. Typhimurium colonization in a 3-D model of colonic epithelium. PLoS One 7:e37116. doi: 10.1371/journal.pone.0037116

Deptula, P., Laine, P. K., Roberts, R. J., Smolander, O.-P., Vihinen, H., Piironen, V., et al. (2017). De novo assembly of genomes from long sequence reads reveals uncharted territories of Propionibacterium freudenreichii. BMC Genomics 18:790. doi: 10.1186/s12864-017-4165-9

Falentin, H., Deutsch, S.-M., Jan, G., Loux, V., Thierry, A., Parayre, S., et al. (2010). The complete genome of Propionibacterium freudenreichii CIRMBIA1T, a hardy actinobacterium with food and probiotic applications. PLoS One 5:e11748. doi: 10.1371/journal.pone.0011748

Ferlez, B., Sutter, M., and Kerfeld, C. A. (2019). Glycyl radical enzyme-associated microcompartments: redox-replete bacterial organelles. mBio 10, e2327-18.

Greening, C., and Lithgow, T. (2020). Formation and function of bacterial organelles. Nat. Rev. Microbiol. 18, 677-689.

Hojo, K., Yoda, N., Tsuchita, H., Ohtsu, T., Seki, K., Taketomo, N., et al. (2002). Effect of ingested culture of Propionibacterium freudenreichii ET-3 on fecal microflora and stool frequency in healthy females. Biosci. Microflora 21, 115120. doi: 10.12938/bifidus1996.21.115

Hosseini, E., Grootaert, C., Verstraete, W., and Van de Wiele, T. (2011). Propionate as a health-promoting microbial metabolite in the human gut. Nutr. Rev. 69, 245-258. doi: 10.1111/j.1753-4887.2011.00388.x
Huang, Y., and Adams, M. C. (2004). In vitro assessment of the upper gastrointestinal tolerance of potential probiotic dairy propionibacteria. Int. J. Food Microbiol. 91, 253-260. doi: 10.1016/j.ijfoodmicro.2003.07.001

Jakobson, C. M., and Tullman-Ercek, D. (2016). Dumpster diving in the gut: bacterial microcompartments as part of a host-associated lifestyle. PLoS Pathogens 12:e1005558. doi: 10.1371/journal.ppat.1005558

Kaneko, T., Mori, H., Iwata, M., and Meguro, S. (1994). Growth stimulator for bifidobacteria produced by Propionibacterium freudenreichii and several intestinal bacteria. J. Dairy Sci. 77, 393-404. doi: 10.3168/jds.s0022-0302(94) 76965-4

Kerfeld, C. A., Aussignargues, C., Zarzycki, J., Cai, F., and Sutter, M. (2018). Bacterial microcompartments. Nat. Rev. Microbiol. 16, 277-290.

Korbel, J. O., Doerks, T., Jensen, L. J., Perez-Iratxeta, C., Kaczanowski, S., Hooper, S. D., et al. (2005). Systematic association of genes to phenotypes by genome and literature mining. PLoS Biol. 3:e134. doi: 10.1371/journal.pbio.0030134

Lu, J., Boeren, S., De Vries, S., Van Valenberg, H., Vervoort, J., and Hettinga, K. (2011). Filter-aided sample preparation with dimethyl labeling to identify and quantify milk fat globule membrane proteins. J. Prot. 75, 34-43. doi: 10.1016/j.jprot.2011.07.031

Macfarlane, G. T., and Gibson, G. R. (1997). "Carbohydrate fermentation, energy transduction and gas metabolism in the human large intestine," in Gastrointestinal Microbiology: Gastrointestinal Ecosystems and Fermentations, Vol. 1, eds R. I. Mackie and B. A. White (Boston, MA: Springer US), 269-318. doi: 10.1007/978-1-4615-4111-0_9

Meile, L., Le Blay, G., and Thierry, A. (2008). Safety assessment of dairy microorganisms: propionibacterium and Bifidobacterium. Int. J. Food Microbiol. 126, 316-320. doi: 10.1016/j.ijfoodmicro.2007.08.019

Quesada-Chanto, A., Schmid-Meyer, A., Schroeder, A., Carvalho-Jonas, M., Blanco, I., and Jonas, R. (1998). Effect of oxygen supply on biomass, organic acids and vitamin B12 production by Propionibacterium shermanii. World J. Microbiol. Biotechnol. 14, 843-846.

Ravcheev, D. A., Moussu, L., Smajic, S., and Thiele, I. (2019). Comparative genomic analysis reveals novel microcompartment-associated metabolic pathways in the human gut microbiome. Front. Genet. 10:636. doi: 10.3389/fgene.2019.00636

Richter-Dahlfors, A., Ravnum, S., and Andersson, D. (1994). Vitamin B12 repression of the cob operon in Salmonella typhimurium: translational control of the cbiA gene. Mol. Microbiol. 13, 541-553. doi: 10.1111/j.1365-2958.1994. tb00449.x

Roessner, C. A., Huang, K.-x, Warren, M. J., Raux, E., and Scott, A. I. (2002). Isolation and characterization of 14 additional genes specifying the anaerobic biosynthesis of cobalamin (vitamin B12) in Propionibacterium freudenreichii (P. shermanii) The GenBank accession numbers for the sequences reported in this paper are AY033235, AY033236, U13043 and U51164. Microbiology 148, 1845-1853. doi: 10.1099/00221287-148-6-1845

Sampson, E. M., and Bobik, T. A. (2008). Microcompartments for B12-dependent 1, 2-propanediol degradation provide protection from DNA and cellular damage by a reactive metabolic intermediate. J. Bacteriol. 190, 2966-2971. doi: 10.1128/jb.01925-07

Saraoui, T., Parayre, S., Guernec, G., Loux, V., Montfort, J., Cam, A. L., et al. (2013). A unique in vivo experimental approach reveals metabolic adaptation of the probiotic Propionibacterium freudenreichii to the colon environment. BMC Genomics 14:911. doi: 10.1186/1471-2164-14-911

Satomi, K., Kurihara, H., Isawa, K., Mori, H., and Kaneko, T. (1999). Effects of culture-powder of Propionibacterium freudenreichii ET-3 on fecal microflora of normal adults. Biosci. Microflora 18, 27-30. doi: 10.12938/bifidus1996.18.27

Seeliger, S., Janssen, P. H., and Schink, B. (2002). Energetics and kinetics of lactate fermentation to acetate and propionate via methylmalonyl-CoA or acrylyl-CoA. FEMS Microbiol. Lett. 211, 65-70. doi: 10.1111/j.1574-6968.2002.tb11204.x

Smaczniak, C., Li, N., Boeren, S., America, T., Van Dongen, W., Goerdayal, S. S., et al. (2012). Proteomics-based identification of low-abundance signaling and regulatory protein complexes in native plant tissues. Nat. Protocols 7:2144. doi: 10.1038/nprot.2012.129

Sokolovskaya, O. M., Shelton, A. N., and Taga, M. E. (2020). Sharing vitamins: cobamides unveil microbial interactions. Science 369:eaba0165. doi: 10.1126/ science.aba0165 
Sutter, M., Greber, B., Aussignargues, C., and Kerfeld, C. A. (2017). Assembly principles and structure of a 6.5-MDa bacterial microcompartment shell. Science 356, 1293-1297. doi: $10.1126 /$ science.aan3289

Thiennimitr, P., Winter, S. E., Winter, M. G., Xavier, M. N., Tolstikov, V., Huseby, D. L., et al. (2011). Intestinal inflammation allows Salmonella to use ethanolamine to compete with the microbiota. Proc. Natl. Acad. Sci. U.S.A. 108, 17480-17485. doi: 10.1073/pnas.1107857108

Toraya, T., Honda, S., and Fukui, S. (1979). Fermentation of 1, 2-propanediol and 1, 2-ethanediol by some genera of Enterobacteriaceae, involving coenzyme B12dependent diol dehydratase. J. Bacteriol. 139, 39-47. doi: 10.1128/jb.139.1.3947.1979

Tyanova, S., Temu, T., Sinitcyn, P., Carlson, A., Hein, M. Y., Geiger, T., et al. (2016). The Perseus computational platform for comprehensive analysis of (prote)omics data. Nat. Methods 13, 731-740. doi: $10.1038 /$ nmeth.3901

Vizcaíno, J. A., Csordas, A., del-Toro, N., Dianes, J. A., Griss, J., Lavidas, I., et al. (2016). 2016 update of the PRIDE database and related tools. Nucleic Acids Res. 44(D1), D447-D456.

Wendrich, J. R., Boeren, S., Möller, B. K., Weijers, D., and De Rybel, B. (2017). In Vivo Identification of Plant Protein Complexes Using IP-MS/MS Plant Hormones. Berlin: Springer, 147-158.

Wilson, J. W. (2021). Manipulating microcompartment operons to study mechanism and function. Curr. Opin. Microbiol. 60, 66-72. doi: 10.1016/j.mib. 2021.01.014
Wiśniewski, J. R., Zougman, A., Nagaraj, N., and Mann, M. (2009). Universal sample preparation method for proteome analysis. Nat. Methods 6:359. doi: 10.1038/nmeth.1322

Xue, J., Murrieta, C. M., Rule, D. C., and Miller, K. W. (2008). Exogenous or L-rhamnose-derived 1, 2-propanediol is metabolized via a pduD-dependent pathway in Listeria innocua. Appl. Environ. Microbiol. 74, 7073-7079. doi: 10.1128/aem.01074-08

Yu, G., Wang, L.-G., Han, Y., and He, Q.-Y. (2012). clusterProfiler: an R package for comparing biological themes among gene clusters. Omics J. Integr. Biol. 16, 284-287. doi: 10.1089/omi.2011.0118

Zeng, Z., Smid, E. J., Boeren, S., Notebaart, R. A., and Abee, T. (2019). Bacterial microcompartment-dependent 1,2-Propanediol utilization stimulates anaerobic growth of listeria monocytogenes EGDe. Front. Microbiol. 10:2660. doi: $10.3389 /$ fmicb. 2019.02660

Conflict of Interest: The authors declare that the research was conducted in the absence of any commercial or financial relationships that could be construed as a potential conflict of interest.

Copyright (c) 2021 Dank, Zeng, Boeren, Notebaart, Smid and Abee. This is an openaccess article distributed under the terms of the Creative Commons Attribution License (CC BY). The use, distribution or reproduction in other forums is permitted, provided the original author(s) and the copyright owner(s) are credited and that the original publication in this journal is cited, in accordance with accepted academic practice. No use, distribution or reproduction is permitted which does not comply with these terms. 